\title{
LA VEILLE NÉOLOGIQUE EN TANT QUE DÉMARCHE À VISÉE LEXICOGRAPHIQUE : EXEMPLE DE L'EMPRUNT RÉCENT À L'ANGLAIS EN FRANÇAIS ET EN POLONAIS
}

Si la fin du $\mathrm{XX}^{\mathrm{e}}$ et le début du $\mathrm{XXI}^{\mathrm{e}}$ siècles apparaissent comme une époque particulièrement favorable pour toute sorte de productions lexicographiques, il reste indiscutable que le niveau de celles-ci est variable, avec des ouvrages d'excellente qualité et d'autres qui sont médiocres. Parmi les défauts majeurs qui semblent concerner même certains dictionnaires sérieux, il convient de noter avant tout celui du statut qu'ils réservent aux néologismes et, par conséquent, à leur intégration. Pourtant, dans le monde actuel où l'échange d'informations s'effectue à une vitesse inconnue auparavant, les langues et leurs lexiques s'enrichissent à un rythme particulièrement rapide, ce qui demanderait d'être reflété aussi rapidement que possible par la lexicographie. Or l'actualisation d'un dictionnaire est un travail gigantesque qui ne saurait être uniquement laissé aux soins des lexicographes. En effet, les listes de mots nouveaux sont souvent dressées par des lexicologues s'occupant de la néologie, grâce à la démarche de veille néologique qui consiste à repérer, inventorier et analyser les nouvelles apparitions lexicales. Son idée est liée à la pratique de l'aménagement terminologique, fruit de la politique linguistique menée par différents gouvernements, notamment en ce qui concerne la langue française (par exemple au Québec, mais aussi en France). À ses sources, cette préoccupation consistait à proposer des termes francophones équivalents à ceux qui avaient été auparavant créés et propagés en anglais, et à suivre la diffusion de ces recommandations grâce à des enquêtes. Plusieurs travaux de terminologie ont été depuis écrits à ce sujet, parmi lesquels il convient de citer, comme 
l'un des premiers, l'article de Pierre Arnaud et Philippe Thoiron consacré à l'évaluation quantitative des termes appartenant aux textes scientifiques ${ }^{1}$. Plus tard, l'ouvrage de Loïc Depecker et Gina Mamavi $(1997)^{2}$, intitulé La mesure des mots. Cinq études d'implantation terminologique, a apporté déjà une synthèse des premières grandes données statistiques sur l'implantation de termes dans le contexte de l'aménagement linguistique. Ce recueil comprenant cinq études réalisées en France de 1991 à 1993 par différentes équipes de recherche a fait état de la vitalité de plusieurs termes précédemment publiés par le Journal officiel. Les analyses de ce type ne sont d'ailleurs devenues possibles que grâce à l'apparition de grands corpus informatiques. Ainsi, dès 2005, Anne Condamines tout en constatant des perspectives importantes de telles études relatives à la terminologie, s'est-elle aussi posé la question de leur utilité éventuelle en linguistique :

On le voit, les perspectives en termes de besoins, d'outils, de méthodes en lien avec l'analyse de corpus, en particulier lorsqu'elle est appliquée à la terminologie, sont très importants. Qu'en est-il d'une vision plus linguistique sur ce même sujet ? ${ }^{3}$

En continuant cette même réflexion, plus récemment, Charlotte Coy $(2012)^{4}$ constatait l'impact des recommandations officielles des Commissions de Terminologie sur la langue commune et son lexique. C'est ainsi, entre autres, que s'est élargi le champ d'application de la veille néologique qui, aujourd'hui, prend en considération non seulement les terminologies spécialisées, mais aussi le vocabulaire commun. Notons enfin plusieurs travaux de Jean-François Sablayrolles qui évoque la possibilité d'aller " au-delà de la simple collecte pour constituer le répertoire des néologismes retenus comme un outil de travail et d'analyse »" grâce, notamment, à « la base Neologia, dictionnaire de néologismes du français contemporain, élaboré au LDI (...) »5. Le repérage de néologismes ne constitue donc que le début du travail qui doit se poursuivre afin de donner leur description la plus complète possible.

Notre article se propose de présenter un exemple particulier de ce type de démarche entreprise par un groupe international de chercheurs réunis au sein du

${ }^{1} \mathrm{P}$. Arnaud et Ph. Thoiron, « Analyse quantitative des textes scientifiques », [dans :] P.J.L. Arnaud et Ph. Thoiron (dir.), Aspects du vocabulaire - Travaux CRTT, Presses Universitaires de Lyon, Lyon 1993, pp. 133-146.

${ }^{2}$ L. Depecker et G. Mamavi (dir.), La mesure des mots. Cinq études d'implantation terminologique, Publications de l'Université de Rouen, Rouen 1997.

3 A. Condamines, «Linguistique de corpus et terminologie », Langages 157, La terminologie : nature et enjeux, 2005, p. 49.

${ }^{4} \mathrm{Ch}$. Coy, «Les recommandations officielles des Commissions de Terminologie et leur rapport à la langue commune : indices pour un profil lexicologique du français », Neologica 5, 2012, pp. 161-173.

5 J.-F. Sablayrolles, « Neologia : un dictionnaire néologique sous forme de base de données », [dans :] S.A. Marcelino Cardoso, S. Mejri et J. Andrade Mota (dir.), Journée des dictionnaires : Les Dictionnaires : ressources, méthodes et nouvelles technologies, Nov 2010, Salvador, Bahia, Brazil. Instituto de Letras da Universidade federal da Bahia, 2011, pp. 221-235. 
projet EMPNEO ${ }^{6}$ dont les débuts datent de 2011. Il a ceci de particulier que son programme de recherche envisage de prendre en considération quatre langues simultanément, le français, le grec, le polonais et le tchèque ${ }^{7}$, et prévoit un travail de veille néologique prenant en compte des emprunts récents, appartenant à la langue générale. Le travail comporte plusieurs phases, dont la première consiste à relever de nouveaux emprunts, le champ de recherche étant essentiellement constitué par les médias. Les mots repérés de cette façon sont répertoriés au moyen de fiches modèles ${ }^{8}$, communiquées ensuite aux autres équipes afin que celles-ci vérifient la diffusion des mêmes lexies dans leurs langues respectives. La phase suivante consiste à comparer la circulation des unités décrites dans chacune des langues représentées. Notons que pour tester le projet et la méthode au début du fonctionnement du groupe, six emprunts ont été choisis, à savoir : chick flick ('type de film à destination d'un public féminin'), cloud computing ('modèle de traitement informatique consistant en l'utilisation de services fournis par des serveurs distants'), finisaż ('clôture solennelle d'une exposition'), free mover ('étudiant réalisant ses études à l'étranger à titre individuel, en dehors des programmes d'échange internationaux'), go fast ('transport rapide de tonnes de marchandise, souvent des drogues, en voitures rapides'), home jacking ('vol de véhicule commis après avoir dérobé les clés dans une habitation, avec ou sans recours à la violence') et soumis ensuite à la description et à une analyse comparative dont les résultats ont été présentés dans la première publication commune du groupe, en $2012^{9}$.

${ }^{6}$ Le projet a réuni dès le début : les membres du laboratoire LDI, J.-F. Sablayrolles (Université Paris 13), John Humbley (Université Paris 7), Christine Jacquet-Pfau (Collège de France) et Camille Martinez (Université de Cergy), Anna Anastassyadi et Georgette Nikolaou (Université Aristote de Thessalonique), Zuzana Hildenbrandt (Université d'Olomouc), Alena Podhorna (Université Masaryk de Brno), Anna Bochnakowa (Université Jagellonne de Cracovie), Alicja Kacprzak, Anna Bobińska et Andrzej Napieralski (Université de Łódź).

${ }^{7}$ Cet article, faute de place, se limite à présenter quelques résultats obtenus par le groupe seulement par rapport à la langue française et polonaise.

${ }^{8}$ Voici la description de la fiche modèle provenant du premier article publié par le groupe : « La fiche se présente sous forme de trois tableaux. Le premier contient la liste des équivalents néologiques, des anciennes dénominations concurrencées et une définition. Les deux autres, bâties sur la même structure, donnent des informations sur l'emprunt et sur l'équivalent (ce dernier tableau peut être démultiplié s'il y a plusieurs équivalents) : présence dans des dictionnaires traditionnels ou des dictionnaires sur la toile, quelques contextes significatifs avec leurs références, des mesures de diffusion et d'acclimatation (informations livrées par consultation de moteurs de recherches, présence dans archives de presse, existence d'une famille dérivationnelle et éventuelles adaptations phoniques, graphiques et morphologiques). Une plage d'informations complémentaires et / ou de commentaires figure après les tableaux. » (A. Anastassyadi, A. Kacprzak, A. Podhorna, J.-F. Sablayrolles, «Emprunts et équivalents : étude de leurs diffusions respectives dans plusieurs langues », Cahiers de Lexicologie 101, 2012, pp. 197-207).

9 Ibidem. 
La première constatation obtenue grâce à cette étude a été unanime : même si, selon le protocole de recherche adopté par le groupe, l'attention est portée à tout emprunt, quelle que soit son origine, en réalité, dans la grande majorité des cas, ce sont des anglicismes, voire plus particulièrement des américanismes, qui sont relevés en masse, les autres langues étant très peu représentées ${ }^{10}$. À l'époque de la mondialisation liée dans une grande mesure à la puissance économique des États-Unis, ce fait n'est pas surprenant ; néanmoins, il semble toujours aussi intéressant d'étudier le phénomène en question de plus près, ceci afin de relever des régularités possibles dans l'apparition de ces néologismes dans différentes langues, notamment en ce qui concerne leur foisonnement dans certains domaines, les parties du discours concernées et les types d'assimilations subies lors du processus d'emprunt.

La deuxième constatation importante résultant du travail des équipes a été que les emprunts recueillis appartiennent à quelques champs notionnels, dont certains semblent visiblement plus particulièrement productifs, dans toutes les langues prises en considération.

En premier lieu, il s'agit de celui des nouvelles technologies de l'information et de la communication (NTIC), ce qui reste conforme au développement ultra rapide de ce secteur au cours des dernières années. Les lexies relevées se rapportent à plusieurs phénomènes relevant du domaine en question et évoquent entre autres :

— des pratiques liées au travail avec l'ordinateur (printer, boldować);

- des pratiques et comportements des utilisateurs d'Internet (cloud computing, spamować, youtuber);

— des pratiques et comportements des utilisateurs de Facebook (selfie, hejtować, liker);

— des pratiques des utilisateurs de Tweeter (hashtag, tweeter, tweetować);

— des pratiques des utilisateurs de smartphones (checker, follować);

- des pratiques des utilisateurs de jeux sur internet (booster, shippować), etc.

Dans le deuxième cas, il s'agit d'usages sociaux récemment apparus, dont plusieurs semblent constituer un autre signe important de notre époque. Parmi les thématiques qui en relèvent, énumérons avant tout :

— des pratiques sociétales, qu'elles soient positives (crowdfunding, bookcrossing) ou négatives (binge drinking, bashing) ;

— des pratiques criminelles (car jacking, home jacking);

— des conduites érotiques pratiquées via les NTIC (cam girl, fappening), etc.

${ }^{10}$ Une remarque importante s'impose cependant : certaines langues peuvent constituer à un moment déterminé une source particulièrement riche pour un seul champ notionnel, ce qui est le cas du vocabulaire relatif à la mode de cuisines non européennes, notamment asiatiques ou sud-américaines. Voir à ce propos l'article d'A. Bochnakowa et de Z. Hildenbrand « Des néologismes culinaires récents en polonais et en tchèque » [à paraître]. 
En troisième lieu, compte tenu des changements concernant l'organisation du travail, tant à l'échelle mondiale que nationale, il est question de tout ce qui a trait au monde du travail :

- le partage international du travail (outsourcing, payrolling);

- le marché du travail (alternance dating, slashing);

- les modalités et l'espace du travail (open space, coworking);

- la santé liée au travail (workaholisme, burn-out), etc.

Les trois listes citées ci-dessus ne sont certainement pas exhaustives, la recherche en cours de l'équipe EMPNEO montrant encore d'autres fonds d'emprunts néologiques possibles, mais elles indiquent sans doute quelques spécificités thématiques de l'emprunt récent à l'anglais en français et en polonais (valables aussi pour le grec et le tchèque).

L'examen des parties du discours concernées par l'emprunt récent montre à son tour que, dans toutes les langues analysées, la priorité revient, comme c'est habituellement le cas, à la catégorie du nom, quelle que soit la structure du mot emprunté, qui peut être aussi bien simple (hipster) que composé (cam girl). Pour les autres catégories ${ }^{11}$, bien moins représentées, c'est le verbe qui est de loin le plus fréquent. Du fait du caractère morphologique du français et du polonais, les emprunts de ce type sont essentiellement des verbes formés sur des radicaux empruntés à l'aide de terminaisons spécifiques. Les bases sont fournies d'un côté par des verbes (fr. follower, pol. follować < angl. to follow) et de l'autre par des noms (pol. shipować < angl. relationship), y compris des noms propres (fr. youtuber, pol. youtubować < angl. Youtube). En ce qui concerne les rares adjectifs, on constate la présence de ceux qui gardent leur forme d'origine (fr. et pol. cool $<$ angl. cool) et de ceux dont les formes sont modifiées (pol. nobiasty < angl. noob). Parfois, le même adjectif de base donne lieu à deux variantes dans la langue cible (pol. cool et coolowy). Des adverbes, peu fréquents, ont été relevés uniquement en polonais où la base adjectivale anglophone peut être accompagnée d'un morphème adverbial indigène (pol. sweetaśnie < angl. sweet, pol. fejkowo $<$ angl. fake). Il est intéressant de noter que seul le polonais donne lieu à la formation des degrés de comparaison synthétiques (pl. coolowiej 'plus cool') ; le français, en accord avec sa tendance analytique, recourt aux formes composées pour exprimer le comparatif (plus cool, moins cool, aussi cool). Les deux langues notent aussi l'emprunt de quelques interjections typiques de la pop culture américaine (fr. waou!, waouh!, pol. tat! < angl. wow!). Celles-ci, venues par le biais du cinéma et de la bande dessinée, ont été relevées dans les deux langues cibles aussi bien à l'oral qu'à l'écrit.

${ }^{11}$ Pour plus d'informations au sujet des emprunts non nominaux consulter aussi l'article de A. Bobińska, A. Kacprzak et J.-F. Sablayrolles, « Emprunts et équivalents non nominaux nouveaux et récents : verbes, adjectifs, adverbes et interjections » [à paraître]. 
L'intégration des unités lexicales empruntées dans différentes langues n'est évidemment pas un phénomène homogène et une autre remarque qui s'impose est que les langues prises en considération ne retiennent pas toujours les mêmes emprunts. Comme exemple, citons le terme polonais hejtować provenant de l'anglais to hate, ayant d'abord appartenu au vocabulaire de Facebook, mais dont l'utilisation s'est ensuite élargie jusqu'à la langue générale pour désigner l'action d'exprimer, le plus souvent d'une façon publique, une dépréciation profonde envers quelqu'un ou quelque chose. Ce verbe a donné lieu en polonais à plusieurs dérivés, dont notamment les noms d'agent hejter, hejtownik, les adjectifs hejtowany, zahejtowany, ainsi que les verbes préfixés zahejtować, pohejtować. Le français n'a pas (encore ?) emprunté ce mot, ce qui est peut-être lié à la difficulté d'assimiler un mot commençant par un $h$. On peut imaginer aussi que la graphie de l'infinitif potentiel *hater serait trop proche de celle du verbe (se) hâter, ce qui fait peut-être obstacle à son apparition. D'autres exemples sont offerts par plusieurs lexies du domaine du marché du travail : notamment le mot job truck utilisé depuis 2014 en français par exemple à propos du recrutement d'employés par la banque BNP Paribas, qui n'a aucune existence en polonais ; de la même façon, jobbing, qui fonctionne déjà bien en français, n'est noté en polonais que dans des noms propres de firmes. Il en va de même de l'adjectif speed qui en français est souvent employé comme attribut dans le syntagme être speed, mais qui n'apparaît pas en polonais.

Une autre constatation du groupe est que l'intégration des emprunts (du point de vue phonétique, graphique, morphologique) s'opère dans chacune des langues de manière autonome, selon leurs spécificités linguistiques.

En ce qui concerne d'abord les adaptations phonétiques et graphiques, le français semble conserver plus souvent que le polonais la graphie originale qui accompagne la prononciation à l'anglaise. Ainsi, en français, on note systématiquement dans les emprunts récents le maintien des lettres doubles à l'écrit (bookcrossing, speed), le maintien de la lettre -w- (follower, forwarder), le maintien de la diphtongue [ai] (liker, live tweeter), le maintien de la prononciation [i+n] (printer). Le polonais garde aussi le plus souvent la prononciation originale, mais en ce qui concerne la graphie, soit on observe le maintien de la graphie d'origine, que celleci soit conforme aux règles de l'orthographe polonaise (boldować, printować) ou non, dans lequel cas elle devrait entraîner selon les règles polonaises une autre prononciation (checkować [t fekovać], crossować [krosovać]), soit on assiste à une modification graphique qui « conserve » la prononciation à l'anglaise (hejtować, lajkować).

D'un point de vue morphologique, des différences sont aussi notables dans l'adaptation des emprunts, ce qui est lié au caractère synthétique du polonais et analytique du français. On constate notamment une nette tendance du polonais à créer des verbes nouveaux sur les bases empruntées à l'aide des préfixes aspectuels, tels : s-/z(a)-(skrosować, zalajkować), wy- (wyboostować), 
prze-(przeforwardować), et autres. À ce propos, il convient aussi de noter quelques concurrences préfixales, par exemple entre $s$ - et prze-, comme dans sforwardować (sens accompli + résultat) / przeforwardować (sens accompli + direction dans l'espace). Le polonais développe aussi de manière systématique des séries dérivationnelles sur la base des verbes, comme c'est le cas pour des noms d'action et leurs variantes préfixées (boldować / boldowanie / wyboldowanie ; boostować / boostowanie / zboostowanie), des adjectifs déverbaux (hejtowany, lajkowany) et des adjectifs déverbaux préfixés (przeforwardowany, skrosowany). En français, le phénomène de dérivation sur les bases empruntées, même s'il existe, est loin d'être systématique. Il concerne surtout les verbes qui peuvent être formés à l'aide de morphèmes verbaux -iser- (youtubiser $<$ Youtube) ou -er (liker < to like). Parmi les préfixes, le re- itératif semble avoir une certaine vitalité (twitter / retwitter). Quelque régularité est notée aussi dans l'apparition des noms d'agent en -eur (spammer / spammeur ; shipper / shippeur). Les deux langues semblent aussi favorables à la formation du féminin sur la base des noms masculins assimilés. Ainsi, en français, shippeuse apparaît à côté de shippeur, en polonais hejterka à côté de hejter, etc.

Analysés du point de vue sémantique, les nouveaux emprunts sont pour la plupart monosémiques, ce qui résulte sans doute de la spécificité de leur référence ainsi que de leur apparition récente. Les verbes comme youtubować / youtub(is)er, tweetować / tweeter, follować / follower et des noms comme chick flick, desk charing, book crossing et beaucoup d'autres ne présentent qu'un sens, identique d'ailleurs en français et en polonais. Notons cependant la polysémie observée dans certains des cas analysés. Elle résulte de l'existence antérieure de certains mots : tel est le cas de checkować en polonais, qui avant de signifier des opérations relatives à l'utilisation des smartphones (à savoir 'marquer sa présence dans un lieu physique, faire connaitre sa localisation, annoncer ce qu'on fait à un moment donné') a été employé dans la terminologie du poker ('miser sans relancer ou égaliser la mise'), ainsi que pour indiquer la procédure de check-in à l'aéroport. Il en va de même du verbe speedować (ang. speed) qui, en polonais, connaît au moins quatre significations. Il semble qu'à la plus ancienne, la plus proche du mot de base anglais ('se dépêcher, faire vite'), d'autres, plus spécifiques, se soient ensuite ajoutées dans un processus d'extension sémantique ('prendre de l'amphétamine', 'nager rapidement — en parlant des poissons dans un aquarium', 'descendre à ski avec un parapente'). En français, l'emprunt speeder, qui a d'abord pris une forme intransitive, a aussi gardé en premier lieu le sens du mot de base ('faire vite, se dépêcher') ; par contre la forme transitive (ça me speede!) apparue récemment a développé aussi un nouveau sens ('angoisser, énerver') que l'on retrouve par ailleurs dans les adjectifs speed et speedé. Notons à la fin que certains emprunts récents peuvent présenter un sens flou du fait de leur statut sémantique encore instable. C'est le cas par exemple d'un terme polonais qui existe dans deux versions orthographiques, shippować 
ou szipować (angl. < relationship), utilisé surtout par les très jeunes pour parler de personnages de jeux électroniques, voire d'acteurs ou d'autres personnes connues. La syntaxe du verbe est instable, car le verbe se combine tantôt avec le génitif, tantôt avec l'accusatif. Il en va de même de son sens oscillant entre 'soutenir, approuver une relation entre deux personnes' et 'soutenir qqn, qch en général' ; l'évolution sémantique de ce mot reste donc à suivre, ce qui constitue sans doute l'un des défis les plus fascinants des études de néologie.

Cette brève présentation de quelques résultats d'une recherche menée dans le cadre du projet EMPNEO a sans doute montré l'utilité de la démarche de veille néologique, qu'elle soit appliquée à une langue ou à plusieurs. Compte tenu du fait qu'un mot étranger, au moment de son utilisation ponctuelle dans une langue, ne constitue encore à cette phase qu'un candidat à l'intégration ${ }^{12}$, la veille néologique prenant en compte les emprunts semble un instrument performant, permettant de les enregistrer et de suivre leur évolution dans la langue cible.

Ce qui plus est, le repérage et la description des emprunts dans plusieurs langues simultanément rend compte de la dynamique du processus en question et fournit des informations importantes non seulement sur les lexies prises en considération, mais aussi sur le fonctionnement des langues en général, grâce au point de vue contrastif adopté.

Quant aux néologismes collectés grâce à cet instrument de recherche, ils forment sans doute un corpus de données les plus récentes, bien utile pour un lexicologue ; d'autre part, ces « mots en listes » présentent une finalité lexicographique certaine.

\title{
VEILLE NÉOLOGIQUE AS A LEXICOGRAPHIC APPROACH. ILLUSTRATED BY THE EXAMPLE OF NEW BORROWINGS FROM ENGLISH TO FRENCH AND POLISH
}

\author{
Summary
}

While the turn of the 21 st century appears to be the time of a particularly abundant production of many kinds of dictionaries, their quality is without doubt somewhat uneven: amongst them are both works that are outstanding and works that are mediocre. One of the common weaknesses of most language dictionaries is the status that they give to neologisms, including borrowings. It is a serious problem in the modern era of great technological and cultural progress, when nearly every day the discourse is enriched by new words. Lexicography should denote them as soon as possible, but this is rather difficult as updating a dictionary is a time-consuming venture. However, research conducted by lexicologists can be helpful in it.

12 Voir à ce propos : A. Lehmann et F. Martin-Berthet, Introduction à la lexicologie, Nathan, Paris 2000, pp. 4-6. 
The article presents one of the relatively new approaches of lexicological registration and elaboration on neologisms (so called veille néologique, i.e. neological watch). As an example we will present an international project, EMPNEO, which aims to research the spreading of new borrowings, most frequently from English, to some selected languages.

Key words: lexicography, neology, neological watch, borrowing. 